\title{
Formación de Profesores de Matemáticas desde la Etnomatemática: estado de desarrollo*
}

\author{
Mathematics Teacher Training from Ethnomathematics: \\ a developmental state
}

\author{
Hilbert Blanco-Álvarez ${ }^{* *}$ \\ Alicia Fernández-Oliveras ${ }^{* * *}$ \\ María Luisa Oliveras ${ }^{* * * *}$
}

\begin{abstract}
Resumen
Este estudio tiene como objetivo contribuir al conocimiento del estado de desarrollo de la investigación sobre la formación de profesores de matemáticas desde una perspectiva etnomatemática. El material empírico se seleccionó a partir de artículos publicados entre 1995 y 2015. El análisis fue orientado por cuatro preguntas: ¿Qué características debe tener el currículo escolar basado en una perspectiva etnomatemática?, ¿Cuál debe ser el conocimiento didáctico-matemático del profesor para atender dicho currículo?; ¿Qué características tienen los cursos de formación inicial y continua desde una perspectiva etnomatemática?; y ¿Qué marcos teóricos y metodologías utilizaron las investigaciones? Concluimos que el profesor debe tener un perfil creativo, reflexivo, investigador, capaz de desarrollar un currículo abierto a otras racionalidades; los cursos de formación versan sobre la naturaleza de las matemáticas y el diseño de actividades conectadas con la cultura, y las metodologías son un gran aporte a la investigación cualitativa-interpretativa. Proponemos un modelo sobre el desarrollo profesional del profesor y sus relaciones con otros elementos del sistema educativo, y una estructura para un curso de formación de profesores desde la Etnomatemática.
\end{abstract}

Palabras claves: Formación de Profesores de Matemáticas. Etnomatemática. Currículo Cultural. Estado del Arte.

\begin{abstract}
The goal of this study is to contribute to our understanding of the state of research development about math professors' training from an ethnomathematics perspective. The empirical material was selected from articles published between 1995 and 2015. The analysis was oriented around four questions: What characteristics should

\footnotetext{
* Los resultados que se presentan en este artículo, hacen parte de la investigación doctoral titulada Elementos para la formación de profesores de matemáticas desde la Etnomatemática dirigida por las Dras. María Luisa Oliveras del Grupo de Investigación Etnomatemáticas, Formación de Profesores y Didáctica y Alicia FernándezOliveras del Departamento de Didáctica de las Ciencias Experimentales, ambas de la Universidad de Granada, España.

** Doctorando en Educación por la Universidad de Granada (UGR), Granada, España y Docente de la Universidad de Nariño (UDENAR), Pasto, Nariño, Colombia. Dirección Postal: Facultad de Ciencias Exactas y Naturales, Universidad de Nariño, Calle 18 Carrera 50, Ciudadela Universitaria Torobajo, Pasto, Nariño, Colombia, CP. 52001. E-mail: hilbla@udenar.edu.co.

*** Doctora en Física por la Universidad de Granada (UGR). Investigadora y Profesora de Didáctica de las Ciencias Experimentales en la Universidad de Granada, Granada, España. Dirección Postal: Campus Cartuja s/n 18079. Granada, España. Email: alilia@ugr.es.

***** Doctora en Matemáticas por la Universidad de Granada (UGR). Investigadora y Catedrática de Didáctica de la Matemática en Universidad de Granada (UGR), Granada, España. Dirección Postal: Campus Cartuja s/n 18079. Granada. España. E-mail: oliveras@ugr.es.
} 
a school curriculum based on an ethnomathematics perspective have? What should the didactic-mathematic knowledge of the professor be in order to attend to such a curriculum? What characteristics do the initial and continuing training courses from an ethnomathematics perspective have? and What theoretical and methodological and theoretical frameworks were used in the investigations? We concluded that the professor must have a creative and reflexive profile, be a researcher, and someone capable of developing a curriculum open to other rationalities; the formation courses teach about the nature of mathematics and the design of activities connected to culture and the methodologies are an important contribution to qualitative-interpretive research. We propose a model about the professional development of the professor and his/her relationship to other elements of the educational system, as well as a structure for a professor formation course from an Ethnomathematics perspective.

Keywords: Mathematics Teachers Education. Ethnomathematics. Cultural Curriculum. State of the Art.

\section{Introducción}

La investigación sobre formación de profesores de matemáticas ha venido en aumento, desde hace al menos dos décadas, y ha ganado espacios en las agendas internacionales de la Educación Matemática. Prueba de ello es la literatura que se encuentra (BALL, 2000; CARRILLO, 2011; DOMITE, 2012; GODINO et al., 2013; OLIVERAS; GAVARRETE, 2012; PONTE, 2012, 2013; SHULMAN, 1986; entre otros), cada vez más numerosa y un número creciente de tesis doctorales (ALBANESE, 2014; GAVARRETE, 2012; OLIVERAS 1996; ROJAS, 2014; RAMOS, 2014; entre otros), así como la presencia de esta línea de investigación en medios de difusión específicos que son referentes internacionales como The International Handbook of Mathematics Teacher Education (TIROSH; WOOD, 2008) y Third International Handbook of Mathematics Education (CLEMENTS et al., 2013).

De tan amplia literatura, este estudio tiene como finalidad realizar un análisis de los artículos, que se centran en la formación de profesores de matemáticas desde la Etnomatemática ${ }^{1}$, publicados entre 1995 y 2015 en revistas del área.

$\mathrm{El}$ análisis fue orientado por cuatro preguntas que surgieron al estudiar el conjunto de los artículos: 1) ¿Qué características debe tener el currículo escolar orientado desde una perspectiva etnomatemática?, 2) ¿Cuál debe ser el conocimiento didáctico-matemático ${ }^{2}$ del profesor para desarrollarlo?; 3) ¿Qué características tienen los cursos de formación inicial y continua en relación a los objetivos, la metodología, los contenidos y la evaluación?; y 4) ¿Qué marcos teóricos y qué metodologías se utilizaron en la investigación?

\footnotetext{
${ }^{1}$ En adelante nos referiremos a Etnomatemática (con mayúscula y singular) cuando nos referimos al campo de investigación y etnomatemáticas (con minúscula y plural) cuando nos referimos a las distintas prácticas matemáticas de un grupo cultural o a aspectos educativos.

${ }^{2}$ El conocimiento didáctico-matemático del profesor se caracteriza a partir de tres dimensiones: dimensión matemática, dimensión didáctica y dimensión meta didáctico-matemática (PINO-FAN; GODINO, 2015).
} 


\section{Metodología}

Esta investigación es de tipo documental ${ }^{3}$ (GIL, 1999), desarrollada con metodología cualitativa e interpretativa, hizo uso de una técnica de análisis de contenido ${ }^{4}$ (BARDIN, 1996).

\subsection{Tipos de materiales y método de búsqueda}

La recolección del material empírico se centró en artículos de revistas científicas publicados entre 1995 y 2015. La búsqueda de éstos se realizó en dos grupos de bases de datos. El primer grupo compuesto por las bases de datos Web of Science, Scopus y Springer; el segundo grupo compuesto por las bases de datos Redalyc, Scielo y Google scholar.

Los terminos de búsqueda usados en ambos grupos fueron: teacher education and ethnomathematics; teacher training and ethnomathematics; in-service teacher education and ethnomathematics; pre-service teacher and ethnomathematics y sus respectivas traducciones al español y portugués.

\subsection{Material recolectado}

En total encontramos 38 artículos, como se muestra en la Tabla 1. Además, encontramos 14 capítulos de libros, 35 ponencias en congresos, 3 tesis doctorales, y 10 trabajos de fin de máster, sin embargo, para fines de este artículo analizamos solo los artículos.

Tabla 1 - Listado de revistas y número de artículos publicados en los periodos 1995-2005 y 2006-2015

\begin{tabular}{|l|c|c|}
\hline \multicolumn{1}{|c|}{ Revistas } & $\mathbf{1 9 9 5 - 2 0 0 5}$ & $\mathbf{2 0 0 6 - 2 0 1 5}$ \\
\hline Asia-Pacific Journal of Teacher & & 1 \\
\hline Bolema: Boletim de Educação Matemática & & 2 \\
\hline Journal of Mathematics and Culture & & 3 \\
\hline Journal of Mathematics Teacher Education & & \\
\hline RELIME: Revista Latinoamericana de Investigación en Matemática Educativa & & 1 \\
\hline REDIMAT: Journal of Research in Mathematics Education & 1 & 1 \\
\hline Revista Discursos & & 1 \\
\hline Revista Educação Matemática em foco & & 1 \\
\hline Revista Educación y Pedagogía & & 1 \\
\hline Revista Horizontes & & \\
\hline
\end{tabular}

\footnotetext{
${ }^{3}$ La investigación documental hace uso de los materiales publicados que no han recibido un tratamiento analítico, o que todavía puede ser vuelto a trabajar de acuerdo con los objetivos de búsqueda (GIL, 1999).

${ }^{4} \mathrm{El}$ análisis de contenido visto como un conjunto de técnicas de análisis que emplea procedimientos sistemáticos y objetivos de descripción del contenido del instrumento de registro (BARDIN, 1996).
} 


\begin{tabular}{|c|c|c|}
\hline Revistas & 1995-2005 & 2006-2015 \\
\hline Revista Latinoamericana de Etnomatemática & & 15 \\
\hline Revista Quadrante & 1 & \\
\hline The Journal of Mathematical Behavior & & 1 \\
\hline Uno: Revista de Didáctica de las Matemáticas & 3 & \\
\hline ZDM: The International Journal on Mathematics Education & 2 & 2 \\
\hline TOTAL: 38 & 9 & 29 \\
\hline
\end{tabular}

Fuente: Elaborado por los autores

En la Tabla 1, se aprecia un aumento en el número de artículos publicados entre 2006 y 2015 sobre la formación de profesores de matemáticas desde una perspectiva etnomatemática. Hecho que interpretamos como un creciente interés de los investigadores por comprender el aporte de la Etnomatemática al conocimiento didáctico-matemático y al desarrollo profesional de los profesores de matemáticas en formación y en ejercicio, además evidencia la juventud de esta línea de investigación.

\subsection{Categorías de análisis}

Después de recoger la información, pasamos a clasificarla, de acuerdo con el propósito de los artículos, en tres dimensiones que emergieron al leer todo el material y que se denominaron: epistémica, práctica e investigativa. Las dimensiones y su descripción se muestran en el Cuadro 1.

\begin{tabular}{|l|l|}
\hline \multicolumn{1}{|c|}{ Dimensiones emergentes } & \multicolumn{1}{|c|}{ Descripción del propósito de los artículos } \\
\hline Epistémica & $\begin{array}{l}\text { Artículos que presentan reflexiones teóricas sobre la formación inicial y } \\
\text { continua de profesores de matemáticas, desde una perspectiva } \\
\text { etnomatemática. }\end{array}$ \\
\hline Práctica & $\begin{array}{l}\text { Artículos que describen propuestas y/o desarrollo de experiencias de cursos } \\
\text { de formación inicial o continua de profesores de matemáticas, desde una } \\
\text { perspectiva etnomatemática. }\end{array}$ \\
\hline Investigativa & $\begin{array}{l}\text { Artículos que reportan resultados de investigaciones sobre formación de } \\
\text { profesores desde una perspectiva etnomatemática. }\end{array}$ \\
\hline
\end{tabular}

Cuadro 1 - Dimensiones emergentes y su descripción Fuente: Elaborado por los autores

A continuación, se realizó un proceso de codificación abierta y de comparación constante (STRAUSS; CORBIN, 2002) de las unidades de información correspondientes a los distintos apartados de los artículos que pertenecen a cada una de las dimensiones emergentes del Cuadro 1. Dicho proceso de codificación fue realizado apoyándonos en el uso de Atlas.ti, versión 6.2. Finalmente, las categorías emergentes a las que llegamos y que guiaron el análisis se presentan en el Cuadro 2.

\begin{tabular}{|l|l|l|l|}
\hline $\begin{array}{c}\text { Dimensiones } \\
\text { emergentes }\end{array}$ & $\begin{array}{c}\text { Categorías } \\
\text { emergentes }\end{array}$ & $\begin{array}{c}\text { Subcategorias } \\
\text { emergentes }\end{array}$ & Descripción \\
\hline Epistémica & Características del & Currículo & Se refiere a las características que deben tener el \\
\hline
\end{tabular}




\begin{tabular}{|c|c|c|c|}
\hline \multirow{2}{*}{$\begin{array}{l}\text { (Reflexiones } \\
\text { teóricas sobre } \\
\text { formación de } \\
\text { profesores) }\end{array}$} & \multirow{2}{*}{$\begin{array}{l}\text { currículo escolar y } \\
\text { del conocimiento } \\
\text { didáctico- } \\
\text { matemático del } \\
\text { profesor }\end{array}$} & escolar & currículo escolar desde la Etnomatemática \\
\hline & & $\begin{array}{l}\text { Conocimiento } \\
\text { didáctico- } \\
\text { matemático del } \\
\text { profesor }\end{array}$ & $\begin{array}{llcc}\text { Hace alusión al conocimiento } & \text { didáctico } & \text { y } \\
\text { matemático del } & \text { profesor } & \text { desde } & \text { la } \\
\text { Etnomatemática } & & & \end{array}$ \\
\hline \multirow{4}{*}{$\begin{array}{l}\text { Práctica } \\
\text { (Intervenciones } \\
\text { o propuestas en } \\
\text { formación de } \\
\text { profesores) }\end{array}$} & \multirow{4}{*}{$\begin{array}{l}\text { Características } \\
\text { estructurales de los } \\
\text { cursos de } \\
\text { formación inicial y } \\
\text { continua }\end{array}$} & Objetivos & $\begin{array}{l}\text { Hace referencia a los objetivos planteados en los } \\
\text { cursos de formación inicial o continua de } \\
\text { profesores }\end{array}$ \\
\hline & & Contenidos & $\begin{array}{l}\text { Describe los contenidos estudiados en los } \\
\text { cursos. }\end{array}$ \\
\hline & & $\begin{array}{l}\text { Metodologías de } \\
\text { trabajo }\end{array}$ & $\begin{array}{l}\text { Hace referencia a las metodologías de trabajo } \\
\text { utilizadas en los cursos }\end{array}$ \\
\hline & & Evaluación & $\begin{array}{l}\text { Trata sobre los procesos de evaluación } \\
\text { utilizados en los cursos }\end{array}$ \\
\hline \multirow{2}{*}{$\begin{array}{l}\text { Investigativa } \\
\text { (Informes de } \\
\text { investigación) }\end{array}$} & \multirow{2}{*}{$\begin{array}{l}\text { Características } \\
\text { fundamentales de } \\
\text { las investigaciones }\end{array}$} & Marcos teóricos & $\begin{array}{l}\text { Hace alusión a los marcos teóricos utilizados } \\
\text { para el análisis de datos }\end{array}$ \\
\hline & & Metodologías & $\begin{array}{l}\text { Se refiere a la metodología utilizada en la } \\
\text { investigación de la cual se informa }\end{array}$ \\
\hline
\end{tabular}

Cuadro 2 - Dimensiones, categorías y subcategorías emergentes utilizadas en el análisis. Fuente: Elaborado por los autores

\section{Análisis y resultados}

Se encontraron 38 artículos, de los cuales 13 pertenecen a la dimensión Reflexiones teóricas sobre formación de profesores, 18 a la dimensión Intervenciones o propuestas de formación de profesores, y 7 a la dimensión Informes de investigación.

En el Cuadro 3 presentamos el material encontrado, clasificado de acuerdo con la dimensión a la que pertenece.

\begin{tabular}{|l|l|}
\hline $\begin{array}{c}\text { Dimensiones } \\
\text { emergentes }\end{array}$ & \multicolumn{1}{c|}{ Material recolectado } \\
\hline Epistémica & $\begin{array}{l}\text { Bishop (1995), Blanco-Álvarez (2011), Breda y Do Rosário (2011), D’Ambrosio (2014), } \\
\text { Domite (2010, 2012), Gavarrete (2013), Gerdes (1996, 1998), Moreira (2004), Oliveras } \\
\text { (1999), Rodrigues, Ferreira y Domite (2009), Shirley (2001) }\end{array}$ \\
\hline Práctica & $\begin{array}{l}\text { Formación inicial: Aroca (2010), Fernández-Oliveras y Oliveras (2015), Gavarrete y } \\
\text { Albanese (2015), Gavarrete y Oliveras (2012), Gerdes (2010), Katsap y Silverman (2008), } \\
\text { Naresh (2015), Oliveras (1995), Oliveras y Gavarrete (2012), Santillán (2011). } \\
\text { Formación continua: Albanese, Santillán y Oliveras (2014), Gerdes (2014), Martínez } \\
\text { (2012), Martínez y Oliveras (2015), Mendes (2010), Oliveras (2005), Presmeg (1998), } \\
\text { Santillán y Zachman (2009) }\end{array}$ \\
\hline Iinvestigativa & $\begin{array}{l}\text { Blanco-Álvarez (2012), Breda et al. (2012), Gavarrete (2015), Knijnik y Meregalli (2012), } \\
\text { Madusise (2015), Owens (2014), Verner, Massarwe y Bshouty (2013) }\end{array}$ \\
\hline
\end{tabular}

Cuadro 3 - Dimensiones emergentes y material encontrado

Fuente: Elaborado por los autores

Realizamos el análisis de los artículos pertenecientes a cada una de las tres dimensiones, centrándonos en las categorías presentadas en el Cuadro 2, cuyos resultados mostramos a continuación. 


\subsection{Análisis siguiendo la categoría: características del currículo escolar y del conocimiento didáctico-matemático del profesor}

En adelante se presenta un listado de características enunciadas por diferentes autores para un currículo de matemáticas basado en la cultura y elementos del conocimiento didáctico-matemático del profesor de matemáticas necesarios para llevar a la práctica dicho currículo.

\subsubsection{Subcategoría: currículo escolar}

El currículo escolar, visto desde la Etnomatemática, para diversos autores debe contemplar las siguientes características:

- Reconocer las matemáticas como una construcción humana, social y cultural (BISHOP, 1995; GERDES, 1996; entre otros).

- Admitir que además del pensamiento matemático occidental, del cual históricamente se reconoce su surgimiento en Grecia, existe una amplia diversidad de pensamientos matemáticos en el mundo y otras racionalidades (BISHOP, 1995; SHIRLEY, 2001; entre otros) o multimatemáticas (OLIVERAS, 1999).

- Acrecentar el conocimiento matemático al incorporar matemáticas extraescolares al aula y conocimientos previos de los estudiantes (BLANCO-ÁLVAREZ, 2011; DOMITE, 2012; entre otros).

- Aceptar la existencia de prácticas matemáticas transculturales, como contar, medir, diseñar, localizar, jugar y explicar (BISHOP, 1995).

- Incorporar actividades a partir de las experiencias culturales de los estudiantes y de la comunidad (GAVARRETE, 2013; MOREIRA, 2004; entre otros).

- Promover el respeto, la tolerancia y la equidad a partir del estudio y la reflexión sobre las etnomatemáticas de diversas culturas (GAVARRETE, 2013).

- Reconocer a los estudiantes como recreadores y reconstructores de los conocimientos culturales (BISHOP, 1995).

\subsubsection{Subcategoría: conocimiento didáctico-matemático del profesor}


Para que el profesor de matemáticas desarrolle las ideas presentes en el tipo de currículo expuesto anteriormente, necesita un conocimiento profesional fundamentado en etnomatemáticas.

Este conocimiento profesional podemos caracterizarlo desde el modelo CDM: Conocimiento Didáctico-Matemático a partir de tres dimensiones: dimensión matemática, dimensión didáctica y dimensión meta didáctico-matemática (PINO-FAN; GODINO, 2015).

Utilizaremos el CDM como herramienta analítica ${ }^{5}$ para caracterizar los resultados encontrados y que incluimos en esta subcategoría. Se muestran operativas para este análisis las dimensiones: matemática, didáctica y meta didáctico-matemática, que permiten poner de manifiesto 10 características del conocimiento profesional del profesor, presentes en los documentos analizados y que mostramos a continuación.

\subsubsection{Dimensión matemática ${ }^{6}$}

Dentro de esta dimensión consideramos dos características:

- Característica 1: Estudiar las etnomatemáticas de diversas culturas locales, nacionales e internacionales, en la búsqueda del desarrollo de una conciencia de las matemáticas como un producto sociocultural (GAVARRETE, 2013; GERDES, 1998; SHIRLEY, 2001).

- Característica 2: Promover en el profesor un espíritu de indagación y brindarle la formación necesaria para que sea un profesor-investigador de las etnomatemáticas, de otras lógicas de pensamiento, de otras racionalidades presentes entre sus estudiantes y/o en la comunidad, es una idea compartida por muchos autores como (BREDA; ROSARIO, 2011; OLIVERAS, 1995, 1996, 2005; entre otros).

\subsubsection{Dimensión didactica}

\footnotetext{
${ }^{5}$ Hacemos uso del CDM que se basa en el Enfoque Ontosemiótico EOS puesto que en su componente Epistemológico contempla la naturaleza de las matemáticas desde la perspectiva de Wittgenstein, lo que permite la coexistencia y el diálogo de las matemáticas escolares y las matemáticas extraescolares (OLIVERAS; GODINO, 2015). Una postura diferente sobre la naturaleza de las matemáticas es presentada en los modelos de conocimiento de (BALL; THAMES; PHELPS, 2008) y (CARRILLO; CONTRERAS; FLORES, 2013) donde las matemáticas se refiere exclusivamente a las matemáticas escolares. La aplicación de las dimensiones de este modelo fue posterior al análisis realizado en cada categoría.

${ }^{6}$ En el modelo CDM (PINO-FAN; GODINO, 2015) la dimensión matemática es caracterizada solo por el conocimiento matemático escolar desde dos facetas: común y ampliado. Dicho modelo está en constante cambio y ahora en la dimensión matemática, es también aceptado, por sugerencia del primer autor de este artículo, el conocimiento etnomatemático, como una ampliación de dicha dimensión (GODINO, Comunicación Personal, 23 de febrero de 2016)
} 
Dentro de esta dimensión consideramos características que responden a las diferentes facetas:

- Conocimiento sobre los aspectos cognitivos de los estudiantes (faceta cognitiva) y Conocimiento sobre los aspectos curriculares, contextuales, sociales, políticos, económicos..., que influyen en la gestión de los aprendizajes de los estudiantes (faceta ecológica)

- Característica 3: Colocar el énfasis en los estudiantes, en sus conocimientos previos, en su cultura y en las formas de legitimar sus conocimientos en el aula, así como tender puentes entre los aprendizajes escolares y los extraescolares (BISHOP, 1995; BLANCO-ÁLVAREZ， 2011; BREDA; ROSARIO， 2011; DOMITE， 2012; MOREIRA, 2004).

- Característica 4: Propiciar experiencias al estudiante para que constate que estos conceptos siguen vivos y plenamente contextualizados en las sociedades de hoy en día y que no son, únicamente, un conocimiento caduco (BISHOP, 1995), además que valore el conocimiento extraescolar, en muchos casos oral, de los adultos mayores y encuentre un mayor vínculo de las matemáticas con la vida cotidiana (BLANCOÁLVAREZ, 2011).

- Característica 5: Escuchar al otro, Domite (2012) y Rodrigues, Ferreira y Domite (2009) llaman la atención sobre esta idea basándose en Freire (1996) quien proponía que en términos de aptitud del profesor éste debe estar disponible para escuchar a los estudiantes y abrir su mente hacia la diferencia del pensamiento matemático del otro. En este mismo sentido, Shirley (2001) propone el diálogo entre profesores y estudiantes y entre pares, expresando sus pensamientos e intercambiando ideas entre ellos, reforzando así el aspecto político de las matemáticas y la comunicación.

- Característica 6: Brindar herramientas que le ayuden al profesor a establecer conexiones entre las matemáticas escolares y otras áreas. Shirley (2001) sugiere que la Etnomatemática es una clave para encontrar estas conexiones.

- Característica 7: Ampliar el currículo de formación de profesores de matemáticas, yendo más allá de la literatura en educación matemática, incorporando la Antropología, la Sociología, la Psicología y los resultados de la investigación sobre formación de profesores (DOMITE, 2012), y la Historia de las matemáticas (SHIRLEY, 2001). Incorporar además Historia de las ciencias y de las matemáticas del hombre común (navegantes, militares, curas, comerciantes) en Europa; Historia de 
las etnociencias y de las etnomatemáticas; y la Dinámica de la construcción de nuevo conocimiento (D’AMBROSIO, 2014).

- Característica 8: Re-pensar la escuela como un lugar de encuentro de saberes matemáticos, de culturas, donde se respete la diferencia y se promueva la equidad y la formación de una nueva ciudadanía y no solo como un espacio para la transmisión de conocimientos (MONTEIRO; RODRIGUES MENDES, 2014).

- Conocimiento sobre los recursos y medios que pueden potenciar los aprendizajes de los estudiantes (faceta mediacional):

- Característica 9: Ofrecer al profesor herramientas teóricas y metodológicas que le ayuden a integrar los resultados de la investigación etnomatemática en el diseño de actividades, material didáctico y textos escolares (BLANCO-ÁLVAREZ, 2011; GAVARRETE, 2013; MOREIRA, 2004).

\subsubsection{Dimensión meta didáctico-matemática}

Dentro de esta dimensión consideramos incluida una característica:

- Característica 10: Formar a los profesores como profesionales reflexivos sobre su propia práctica, sobre las necesidades emocionales e intelectuales de los estudiantes y sobre las funciones sociales de la educación y, así, lograr transformaciones en su acción educativa, es lo que proponen Rodrigues, Ferreira y Domite (2009) y Domite (2012) basándose en los trabajos de Schön $(1987,1995)$.

\subsection{Análisis de la categoría: características estructurales de los cursos de formación inicial y continua}

Se presenta un análisis de los elementos utilizados en el diseño y gestión de cursos de formación inicial o continua de profesores de matemáticas que han sido orientados desde una perspectiva etnomatemática. Estos elementos del diseño son: los objetivos, las fases, los contenidos, las metodologías de trabajo utilizadas y la evaluación, que constituyen cada uno de ellos una subcategoría para el análisis de los cursos de formación.

Veamos, entonces, las características que en los artículos estudiados presentan cada una de estas subcategorías.

\subsubsection{Subcategoria: objetivos}


Realizamos una caracterización de los objetivos de los cursos reportados en los artículos de formación inicial o continua y encontramos que son dos los más comunes. Estos son: a) Fomentar en los docentes la reflexión sobre la naturaleza de las matemáticas como una actividad cultural (ALBANESE; SANTILLÁN; OLIVERAS, 2014, NARESH, 2015; entre otros) y b) Desarrollar las capacidades docentes, partiendo del contexto cultural al que pertenece y diseñar actividades para el aula. Actividades ligadas al reconocimiento político de las prácticas extraescolares en el aula (OLIVERAS, 1995; OLIVERAS; GAVARRETE, 2012; SANTILLÁN; ZACHMAN, 2009; MARTÍNEZ, 2012; GERDES, 2010, 2014; SANTILLÁN, 2011; VERNER, MASSARWE; BSHOUTY, 2013; GAVARRETE, 2015; GAVARRETE; ALBANESE, 2015; MARTINEZ; OLIVERAS, 2015; FERNÁNDEZ-OLIVERAS; OLIVERAS, 2015; NARESH, 2015; entre otros). Estos objetivos son de dos estatutos diferentes, el primero es epistemológico, en tanto que busca el estudio y la reflexión de la naturaleza del conocimiento matemático, y el segundo es práctico, en tanto pretende dar al profesor herramientas para la investigación en la cultura y para su aplicación al trabajo en el aula.

En términos de las dimensiones de la Etnomatemática (D’AMBROSIO, 2005) estos objetivos apuntan a fortalecer en los profesores en formación o en ejercicio las dimensiones epistemológica, educacional y política; pues se pretende formar profesores con una concepción amplia de las matemáticas y brindarles las herramientas teóricas y metodológicas para que indaguen en los conocimientos extraescolares de los estudiantes y de la comunidad. De modo que puedan hacer uso de ellos por medio de actividades, buscando no solo un aprendizaje más significativo sino, también, un empoderamiento cultural por parte de los estudiantes y la legitimización de los conocimientos de la comunidad en la escuela.

\subsubsection{Subcategoria: fases}

Los cursos de formación de profesores analizados utilizaron diferentes formas de estructuración. A cada componente de esa estructura le hemos llamado fase. Por ejemplo, en los cursos de formación inicial las fases fueron: estudio de elementos de la cultura, diseño de microproyectos, implementación en el aula, evaluación (OLIVERAS, 1995); investigación sobre un elemento de la cultura, diseño de actividades, socialización (KATSAP; SILVERMAN, 2008); en los cursos de formación continua algunos ejemplos de las fases utilizadas fueron: estudio de la realidad, organización del conocimiento y aplicación del 
conocimiento (MENDES, 2010); estudio de elementos de la cultura y diseño de proyectos (MARTÍNEZ, 2012).

Del estudio de las fases de todos los cursos analizados en los artículos, extraemos unas componentes, en términos de las acciones de cada fase, que constituyen los resultados de esta subcategoría, cuya expresión vemos, como una estructura flexible explicativa y que, a su vez, sirve para el diseño de nuevos cursos de formación de profesores desde la Etnomatemática, que mostramos en el Cuadro 4:

\begin{tabular}{|c|c|}
\hline Fase & Descripción $^{7}$ \\
\hline Estudio teórico & $\begin{array}{l}\text { En esta fase se estudian los fundamentos de la Etnomatemática, se } \\
\text { reflexiona sobre la naturaleza de las matemáticas, se leen artículos de } \\
\text { investigación en Etnomatemática, artículos sobre la integración de la } \\
\text { Etnomatemática al aula escolar, artículos sobre currículo cultural de } \\
\text { matemáticas, etc. }\end{array}$ \\
\hline Estudio de elementos de la cultura & $\begin{array}{l}\text { En esta fase es posible indagar directamente en la comunidad sobre sus } \\
\text { prácticas culturales, o recopilar los saberes que cada uno tenga sobre la } \\
\text { cultura de su comunidad o de otra. También se puede hacer uso de } \\
\text { estudios antropológicos, históricos, arqueológicos, etc. }\end{array}$ \\
\hline Diseño de actividades & $\begin{array}{l}\text { Se analiza la información recolectada en función de su potencial } \\
\text { matemático. Se realiza un análisis didáctico y una transposición didáctica } \\
\text { y se diseñan las actividades, microproyectos, o proyectos. }\end{array}$ \\
\hline Implementación en el aula escolar & $\begin{array}{l}\text { Se implementan las actividades con los estudiantes, prestando especial } \\
\text { interés a la motivación que generan, a los procesos cognitivos y } \\
\text { matemáticos puestos en juego y al valor político (en términos de } \\
\text { legitimación de saberes) de la actividad. }\end{array}$ \\
\hline Evaluación de la implementación & $\begin{array}{l}\text { Se realiza una evaluación de lo sucedido en el aula al poner en juego la } \\
\text { actividad o microproyecto, en términos de los objetivos propuestos, las } \\
\text { dificultades de los estudiantes y del valor político de la actividad. }\end{array}$ \\
\hline Evaluación del curso & $\begin{array}{l}\text { Se realiza una evaluación general del curso que contemple la visión de } \\
\text { las matemáticas de los profesores, el desarrollo del curso, el proceso del } \\
\text { diseño de actividades y la implementación. }\end{array}$ \\
\hline
\end{tabular}

Cuadro 4 - Propuesta de estructura de un curso de formación de profesores desde la Etnomatemática Fuente: Elaborado por los autores

\subsubsection{Subcategoría: contenidos de los cursos}

\footnotetext{
${ }^{7}$ Esta descripción no pretende listar todas las acciones de cada fase, solo se hace a manera de ejemplificar a qué se refiere cada una.
} 
Los contenidos de los cursos son muy variados, algunos de ellos son: a) ejemplos concretos de tecnologías simbólicas que le permitieron a algunas sociedades o grupos sociales hacer procesos de cálculo, jugar, contar etc. de manera diferente a la exclusiva forma del contexto escolar (AROCA, 2010); b) Etnomatemáticas, Enculturación matemática, Microproyectos de educación intercultural basados en Etnomatemáticas (OLIVERAS, 1995, 2005; OLIVERAS; GAVARRETE, 2012; GAVARRETE; ALBANESE, 2015; entre otros); c) construcciones geométricas y estudio de los conceptos matemáticos, multiculturales y didácticos necesarios para la enseñanza de la geometría en el contexto cultural (GERDES, 2010, 2014; VERNER, MASSARWE; BSHOUTY, 2013; entre otros).

De esta forma, al estudiar los fundamentos de la Etnomatemática, se profundiza en las dimensiones histórica, política, educativa, conceptual y epistémológica de la Etnomatemática (D’AMBROSIO, 2005); y al trabajar en los microproyectos y conceptos didácticos necesarios para la enseñanza de la geometría, por ejemplo, en un contexto cultural se potencian las dimensiones educativa, política e histórica.

\subsubsection{Subcategorías: metodologías de trabajo}

Las metodologías de trabajo más comunes que se han utilizado en los cursos de formación realizados por los investigadores estudiados (ALBANESE, SANTILLÁN; OLIVERAS, 2014; AROCA, 2010; FERNÁNDEZ-OLIVERAS; OLIVERAS, 2015; GAVARRETE, 2015; GAVARRETE; ALBANESE, 2015; MARTÍNEZ, 2012; MARTINEZ; OLIVERAS, 2015; OLIVERAS; GAVARRETE, 2012; PRESMEG, 1998; SANTILLÁN, 2011; SANTILLÁN; ZACHMAN, 2009) son: a) trabajo en grupo y reflexiones grupales o grupos de discusión; b) proyectos o microproyectos; c) investigación de prácticas matemáticas o signos culturales en la comunidad; d) creatividad; e) uso y resolución de guías didácticas; f) participación de la comunidad en el aula (AROCA, 2010).

Resaltamos la creatividad como una herramienta potente en la dimensión cognitiva y educativa de la Etnomatemática (D’AMBROSIO, 2005), así como los microproyectos para la investigación de signos culturales en la comunidad por parte de los profesores en formación inicial y continua (OLIVERAS, 1995, 1996, 2006), y la participación de la comunidad en los cursos de formación inicial y continua como una estrategia de la dimensión política de la Etnomatemática.

\subsubsection{Subcategoría: evaluación}


Los instrumentos de evaluación comunes utilizados en los cursos de formación de profesores son: a) trabajos elaborados y presentados durante las atividades didácticas realizadas en el aula (AROCA, 2010; GAVARRETE, 2015; OLIVERAS; GAVARRETE, 2012; entre otros); b) portafolio (OLIVERAS; GAVARRETE, 2012; GAVARRETE, 2015; SANTILLÁN; ZACHMAN, 2009, entre otros); c) autoevaluación y extraevaluación (GAVARRETE, 2015; entre otros) y d) informe final (VERNER; MASSARWE; BSHOUTY, 2013, entre otros).

La evaluación en cursos de formación inicial o continua de profesores pretende ser de tipo formativa, buscando que los profesores generen procesos de reflexión sobre su formación o sobre su práctica; a eso se debe que se privilegie la autoevaluación y el portafolio. Vemos que la tendencia, en la dimensión cognitiva de la Etnomatemática va en la dirección de la reflexión del profesor en formación o en ejercicio y a la autoevaluación de su propio trabajo.

Teniendo en cuenta las ideas presentadas en los apartados 3.1 y 3.2, proponemos un modelo gráfico explicativo, desde nuestra propia interpretación, de los elementos y las interacciones que inciden en la formación y el desarrollo profesional del profesor orientado desde la Etnomatemática.

Se trata de un modelo emergente de nuestro análisis y que contempla las actuaciones del profesor, la comunidad, la Etnomatemática, y el estudiante, con la intención de dejar ver la complejidad inherente a la formación del profesor de matemáticas cuando se orienta desde una perspectiva etnomatemática. Dicho modelo se presenta en el apartado siguiente.

\subsection{Modelo emergente del desarrollo profesional del profesor de matemáticas desde una perspectiva etnomatemática y sus relaciones con otros actores del sistema educativo}

El modelo está configurado por cuatro actores: el profesor, la comunidad ${ }^{8}$, el estudiante y la Etnomatemática, además de las complejas y dinámicas relaciones que se tejen entre ellos en el aula de clase, pero además contempla a dichos actores formando parte de un contexto cultural, social, político, económico y geográfico que los condiciona. Dicho modelo (Figura 1) debe ser estudiado, validado y enriquecido por otros estudios. Veamos cómo concebimos el funcionamiento de dichas relaciones.

\footnotetext{
${ }^{8}$ Otras propuestas de transformación del triángulo didáctico se pueden ver en Tall (1986) que propuso el computador en el cuarto vértice; Rezat y Sträßer (2012) proponen el tetraedro socio-didáctico agregando en el cuarto vértice los artefactos, incluida la tecnología, que están situados cultural, institucional e históricamente, por lo que el dominio social y cultural siempre está incluido; y Jaworski (2012) agrega en el cuarto vértice a los didáctas que trabajarán muy de cerca con el profesor pero sin ninguna conexión a los otros dos vértices.
} 


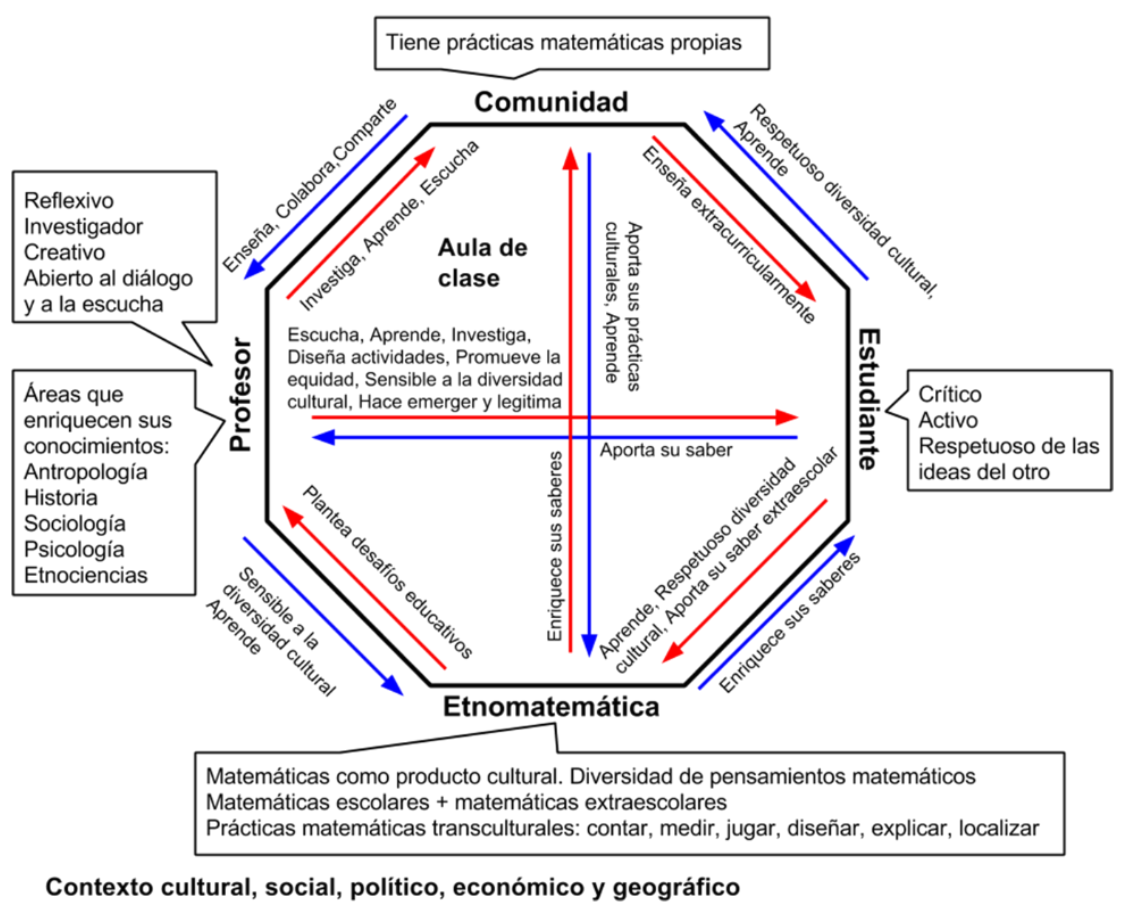

Figura 1 - Relaciones entre el profesor, la comunidad, el estudiante y la Etnomatemática en el aula Fuente: Elaborado por los autores

Primero, el Profesor en su relación con la comunidad, acepta la diversidad cultural, la invita a compartir sus prácticas matemáticas en el aula; el profesor aprende de ella, la escucha, reconoce otras lógicas de razonamiento, otras racionalidades de la comunidad. En cuanto a su relación con la Etnomatemática, él es sensible a la diversidad de prácticas matemáticas, fuera del aula investiga las etnomatemáticas que circulan en las prácticas culturales ampliando, así, su visión de las matemáticas, aprendiendo otras etnomatemáticas, otras historias de las matemáticas, amplia sus concepciones ${ }^{9}$ sobre las matemáticas. Con relación al estudiante, el profesor los escucha, y tiene en cuenta sus conocimientos extraescolares, los cuales legitima y valora políticamente en el aula, aprende de ellos, empoderando así a los estudiantes. Además, diseña actividades teniendo en cuenta las etnomatemáticas de la comunidad, y promueve la equidad y la inclusión. Para todo esto el profesor debe ser reflexivo, creativo, abierto al diálogo y a la escucha, investigador que debe tener en cuenta, para fundamentar sus acciones, áreas como la Antropología, la Sociología, la Psicología, las Historia de las etnomatemáticas, entre otras.

\footnotetext{
${ }^{9}$ Las concepciones como “(...) una estructura más general, incluyendo creencias, significados, conceptos, proposiciones, reglas, imágenes mentales, preferencias y similares. Aunque puede que la distinción no tenga una gran importancia, en ocasiones será más natural referirse a las concepciones de los profesores sobre las matemáticas como disciplina, que hablar simplemente de las creencias de los profesores sobre las matemáticas" (THOMPSON, 1992, p. 130) (...) "Una concepción del profesor sobre la naturaleza de las matemáticas puede verse como creencia, concepto, significado, regla, imagen mental y preferencia, consciente o inconsciente del profesor en relación con las matemáticas. Éstas creencias, conceptos, puntos de vista y preferencias, constituyen los rudimentos de una filosofía de las matemáticas" (Ídem, 1992, p. 132) (traducción nuestra).
} 
Segundo, la Comunidad en relación con el profesor y el estudiante, comparte en el aula y fuera de ella sus etnomatemáticas presentes en sus prácticas culturales. En relación con la Etnomatemática, la comunidad por medio del ejercicio de sus prácticas culturales contribuye a cargar de significado los conocimientos matemáticos y a reconocer y valorar otras racionalidades.

Aunque aquí hemos presentado la incorporación de la comunidad de manera teórica, en la práctica esta relación de la comunidad con los demás actores no es tan inmediata, pues en las sociedades están en juego relaciones de poder que deslegitiman los conocimientos comunitarios en la escuela, estableciendo qué es matemática y qué no, y quitan el poder educativo a la familia y la comunidad, como lo señalan (DÍEZ-PALOMAR, 2011; MORGAN, 1998).

Tercero, el Estudiante en relación al profesor participa con sus conocimientos extraescolares en el aula. En relación con la comunidad, es respectuoso de sus prácticas matemáticas, aprende de ella en el aula y fuera de ella, la representa, respeta y valora los conocimientos de los adultos mayores. En relación a la Etnomatemática, el estudiante aporta sus etnomatemáticas (algoritmos no convencionales de operaciones, patrones de medición no estandarizados, etc.). Este aporte se convierte en un cambio de visión profundo sobre el papel del estudiante en la escuela y en particular lo posiciona como un agente productor de conocimiento y no solo como receptor.

Cuarto, la Etnomatemática concibe las matemáticas como un producto cultural y humano; es un cuerpo de conocimientos conformado por las matemáticas escolares y las matemáticas extraescolares en las prácticas culturales de todo el mundo y las de los pueblos extintos. En relación al profesor, le propone nuevos retos al presentarle la dificultad de intentar transitar desde las práticas culturales a la práctica pedagógica, representada en el diseño de actividades para el aula. Este ejercicio de disciplinarización de las prácticas culturales y de transposición didáctica debe ser analizado en mayor detalle en la formación de profesores desde la perspectiva etnomatemática.

Todas las relaciones representadas en el modelo suceden en el interior del aula, dentro de la escuela, que debe ser repensada como un espacio de reflexión y crítica más que un espacio de transmisión de conocimientos, en el marco de un contexto social, cultural (monocultural, multicultural), político, económico, geográfico (urbano, rural) y educativo (intercultural, bilingüe, multilingüe).

Dicho modelo (Figura 1) guarda relación con: a) el Modelo Didáctico Emergente MED propuesto por Oliveras (1996), en tanto comparten varias de sus características, y b) la 
red de prácticas propuesta por Valero (2012), puesto que señala la importancia y la necesidad de construir agendas de investigación que nos permitan desentrañar la complejidad relacional al analizar la formación de profesores junto a la comunidad, los estudiantes y el conocimiento etnomatemático.

\subsection{Análisis de la categoría: características fundamentales de las investigaciones}

Hemos analizado, aquí, los artículos que presentaban reportes de investigación y centramos la atención en los marcos teóricos utilizados en el análisis de los datos y las metodologías utilizadas.

\subsubsection{Subcategoria: marcos teóricos}

Los marcos teóricos encontrados son: Estructuras de compromiso (GOLDIN et al., 2011) utilizado por Verner, Massarwe y Bshouty (2013) quienes realizaron una investigación en Israel para analizar la motivación en un curso no convencional de formación de profesores desde una perspectiva Etnomatemática. También participaron profesores en formación inicial. Los datos se analizaron con la metodología de las estructuras de compromiso de Goldin et al. en un estudio de casos. Finalmente, proponen una nueva estructura: Reconocer mi cultura, para expresar la motivación que surge de las interacciones multiculturales que fomenta el aprendizaje matemático.

Identidad del pensador matemático (OWENS, 2007) utilizado por Owens (2014) quien presenta los resultados de una investigación realizada en Papua Nueva Guinea donde se analizaron 60 proyectos de matemáticas que tenían una fuerte relación con la cultura. Éstos fueron escritos por profesores en formación y en ejercicio. Se concluye que las relaciones de la cultura y las matemáticas escolares influyen en la identidad del profesor como pensador etnomatemático.

Pedagogía culturalmente responsable (LADSON-BILLINGS, 1996) utilizado en Madusise (2015) quien presenta los resultados de una investigación realizada junto con tres profesores con el objetivo de buscar nuevas formas de enseñar matemáticas conectándola con conceptos claves de la cultura. Se diseñaron actividades, se implementaron y se reflexionó sobre el proceso de intervención en el aula. Se encontró que los profesores se hicieron conscientes de la riqueza y la posibilidad de utilizar las aldeas culturales como recursos 
didácticos para la conexión de la educación matemática con las culturas de los alumnos, en el plan de estudios de Sudáfrica.

Filosofía del lenguaje (WITTGENSTEIN, 1999) y los trabajos de (FOUCAULT, 1979, 2002, 2007) utilizados por Knijnik y Meregalli (2012) quienes presentan los resultados una investigación que tuvo como objetivo comprender las verdades en educación matemática que circulan en los cursos de pedagogía y cómo la diferencia cultural opera en el establecimiento de tales verdades. Se realizaron entrevistas a profesores en ejercicio. Se encontró que el curso de pedagogía permitió a los profesores reflexionar sobre sus experiencias anteriores con las matemáticas escolares; posibilitó la apropiación de juegos de lenguaje asociados a las matemáticas instituidas por diferentes formas de vida; evidenció estereotipos de género en relación con la percepción sobre los estudiantes: los varones obtendrían mejor desempeño en la matemática escolar y las mujeres serían obedientes y capaces de seguir reglas en las aulas de matemáticas.

Las teorias discursivas foucaultianas (FOUCAULT, 1979, 2001, 2002) utilizadas por Breda et al. (2012) para analizar cómo, a través de las singularidades discursivas y de las relaciones de poder-saber, son producidos diferentes modos de subjetivación en los sujetos investigadores. Para esto se analizaron dos trabajos de fin de máster que trabajaron la Etnomatemática en la línea de formación de profesores. Finalmente, se concluye que los discursos de los trabajos de fin de máster analizados, operan como mecanismos de gobierno capaces de habilitar y conducir conductas, produciendo de esta forma, modos singulares de subjetivación.

Las dimensiones de la Etnomatemática (D’AMBROSIO, 2005) utilizadas por BlancoÁlvarez (2012) que presenta un estudio sobre las actitudes hacia la postura sociocultural y política de la educación matemática en estudiantes de grado de educación primaria en la Universidad Autónoma de Barcelona, encontrando que dicha actitud es moderadamente positiva; y Gavarrete (2015) que, además, se basa teóricamente en la Enculturación matemática (BISHOP, 1999), presenta todo el proceso de su tesis doctoral hasta llegar a la construcción de un Curso de Formación Docente basado en Etnomatemáticas Indígenas y su implementación. La evaluación se realizó a través de un modelo etnográfico participativo. Se aportan elementos de discusión acerca de la pertinencia cultural y la formación docente, así como sobre la relación entre el conocimiento cultural y el conocimiento matemático escolar.

Estos marcos teóricos señalan los enfoques que ha seguido la investigación de la formación de profesores de matemáticas desde una perspectiva etnomatemática. 
Haciendo uso del modelo MEDIPSA ${ }^{10}$ (OLIVERAS, 1996) ilustramos, en la Figura 2, el lugar de estos marcos teóricos.

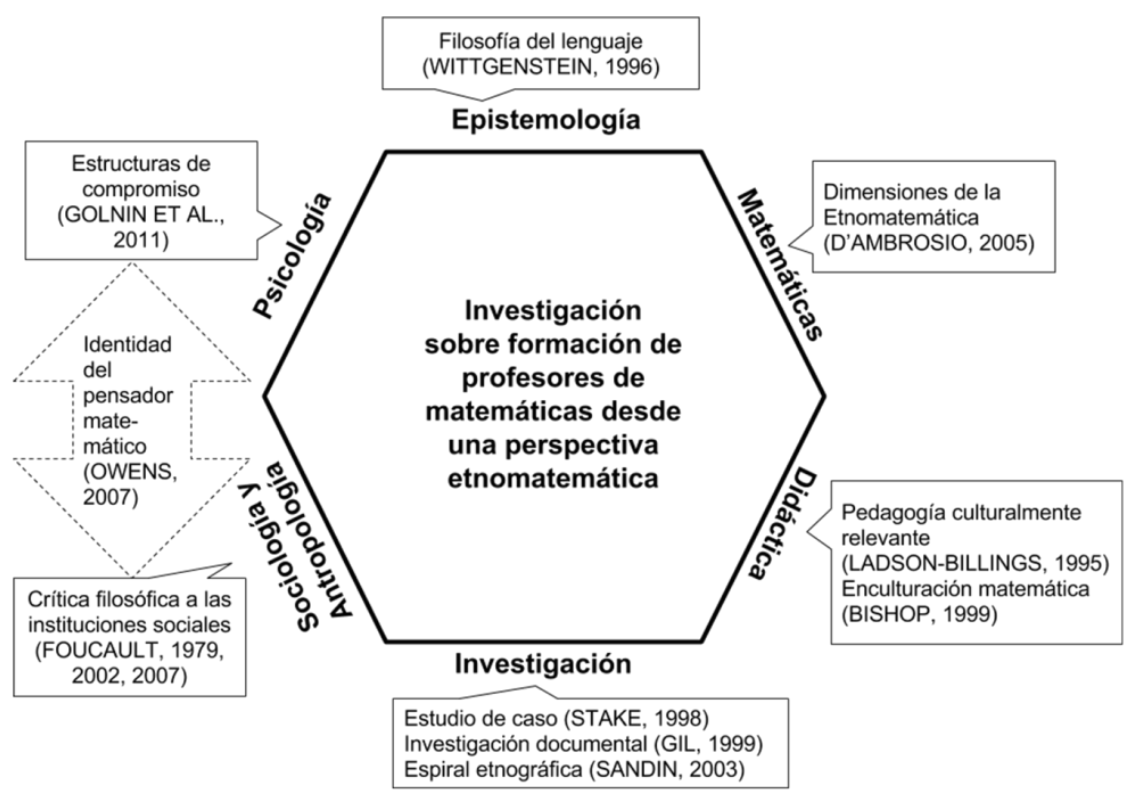

Figura 2 - Marcos teóricos y metodologías ubicados las dimensiones del modelo MEDIPSA Fuente: Elaborado por los autores

\subsubsection{Subcategoria: metodologías}

Encontramos que los seis reportes de investigación se enmarcan en un enfoque cualitativo, interpretativo y que el diseño de la investigación en varios de ellos responde a estudios de caso. Para la recolección de datos se utilizaron instrumentos como la encuesta, entrevistas, actividades escritas de los profesores, videos y grabaciones de discusiones grupales.

Este enfoque metodológico está en consonancia con la naturaleza de la investigación etnomatemática, centrada en el análisis de los procesos de generación, difusión e institucionalización del conocimiento matemático (D’AMBROSIO, 2005) y se ubica en el modelo MEDIPSA en la componente de Investigación, como se muestra en la Figura 2.

\section{Conclusiones}

\footnotetext{
${ }^{10}$ El modelo teórico MEDIPSA es un modelo que fue elaborado por Oliveras (1996), para fundamentar la investigación sobre formación de profesores desde una perspectiva etnomatemática. Es un marco de fundamentos para realizar el análisis e interpretación de los datos. Dicho modelo está formado por distintas teorías que son compatibles entre sí, procedentes de disciplinas que se establecen como constituyentes de las siete componentes del fenómeno didáctico-matemático desde la perspectiva etnomatemática: Matemáticas, Epistemología, Didáctica, Investigación, Psicología, Sociología y Antropología.
} 
Hemos generado tres dimensiones que emergieron al leer todo el material y que se denominaron: epistémica, práctica e investigativa, siendo la práctica a la que pertenecen la mayoría de los trabajos analizados y diversas categorías de análisis que comentamos a continuación.

En la primera categoría Características del currículo escolar y del conocimiento didáctico-matemático del profesor encontramos que la tendencia es hacia la conceptualización de un currículo escolar abierto a otras racionalidades. Con respecto al conocimiento didáctico-matemático del profesor se propende por un profesor reflexivo, creativo, investigador, cuya formación inicial o continua incluya elementos más exigentes que los contenidos, como por ejemplo resultados de la investigación en formación de profesores de matemáticas en general y desde la perspectiva etnomatemática.

En la segunda categoría Características estructurales de los cursos de formación inicial y continua concluimos que los objetivos de los cursos se inclinan hacia dos aspectos del conocimiento didáctico-matemático de los profesores, como son la naturaleza de las matemáticas y el diseño de actividades haciendo uso de elementos de la cultura. Los contenidos, a su vez, repondieron a los objetivos, señalados arriba, y se centraron en contenidos sobre Etnomatemática y Didáctica de las matemáticas. Las metodologías didácticas privilegian el trabajo constructivo y grupal. La implicación social es hacia la inclusión y la equidad al integrar a la comunidad. Las formas de evaluación están orientadas a la formación de profesores reflexivos y autocríticos de su desarrollo profesional.

Además, todos los cursos analizados comparten la participación activa de los profesores y su implicación en su propio aprendizaje, lo que reporta satisfacción, motivación, interés y apropiación por parte de los profesores participantes.

De este análisis concluimos que, a pesar de dotar al profesor de herramientas metodológicas para la investigación etnomatemática y el diseño de actividades en el aula, aún no es claro cómo, cuándo y cuál sería la participación de la comunidad en el aula y cómo llevar a cabo dicha integración en el diseño curricular y en el aula de clase.

Nos surgen, así, nuevas preguntas que formulamos en el marco de agendas de investigación: ¿Cómo se están formando a los futuros profesores o en ejercicio, para la multiculturalidad de las aulas de matemáticas?, ¿cómo lograr las características de un profesor etnomatemático en formación o en ejercicio presentadas en la Figura 1?, ¿cómo acercar la escuela a las prácticas de la comunidad y viceversa?, ¿qué características deben tener los textos escolares para las aulas interculturales? 
Además de estas preguntas, una característica que encontramos en todos los cursos de formación inicial o continua, y que nos preocupa, es que éstos no son permanentes en los currículos de formación de profesores, sino que han respondido a procesos coyunturales de investigación. Shirley (2001) ya había señalado la necesidad y la importancia de integrar dichos cursos en los currículos de los programas de formación inicial de profesores en las universidades. Es importarte señalar que, si bien existen universidades que incluyen en los currículos de formación inicial cursos de Etnomatemática, como los señalados en (BLANCOÁLVAREZ; HIGUITA RAMIREZ; OLIVERAS, 2014; AROCA; BLANCO-ÁLVAREZ; GIL, 2016), no se han publicado investigaciones que describan, caractericen y analicen los aportes de la Etnomatemática en dicha formación.

En la tercera categoría, Características fundamentales de las investigaciones, concluimos que estos reportes de investigación nos presentan marcos teóricos que abren caminos para la interpretación de los datos y que generan nuevos aportes a la formación de profesores desde las perspectivas epistemológica, matemática, didáctica, antropológica y psicológica.

Las metodologías son un gran aporte a la línea cualitativa-interpretativa generando ejemplos de investigaciones que validan este enfoque.

Finalmente, estos análisis nos permitieron, por un lado, plantear un modelo (emergente del estudio realizado) de las relaciones entre el profesor, la comunidad, el estudiante y la Etnomatemática en el aula e inmersos a su vez en un sistema cultural, político, social, económico y geográfico, que brinda elementos a tener en cuenta en la formación inicial y continua de profesores de matemáticas desde una perspectiva etnomatemática.

Por el otro, en relación a las fases de los cursos nos hemos permitido proponer una posible estructura de un curso para la formación de profesores desde la Etnomatemática. Dicha estructura esperamos sea estudiada y puesta en práctica.

Confiamos en que esta revisión ayude a profesores e investigadores a tener una visión de conjunto de lo realizado entre 1995 y 2015 en la investigación sobre la formación de profesores de matemáticas desde una perspectiva Etnomatemática y motive a los investigadores noveles de la mano de los profesores y de la comunidad a iniciarse en esta prolífera línea de investigación.

\section{Agradecimentos}


Agradecemos a Juan D. Godino, María Teresa Castellanos, Aldo Parra, Carolina Tamayo-Osorio, Armando Aroca y Pilar Peña-Rincón por sus comentarios.

\section{Referencias}

ALBANESE, V. Etnomatemáticas en artesanías de trenzado y concepciones sobre las matemáticas en la formación docente. 2014. 223 f. Tesis (Doctorado en Educación) - Universidad de Granada, España. 2014. No publicado.

ALBANESE, V.; SANTILLÁN, A.; OLIVERAS, M. L. Etnomatemática y formación docente: el contexto argentino. Revista Latinoamericana de Etnomatemática, Colombia, v. 7, n. 1, p. 198-220, feb. 2014.

AROCA, A. Una experiencia de formación docente en Etnomatemáticas: estudiantes afrodescendientes del Puerto de Buenaventura, Colombia. Revista Horizontes, Brasil, v. 28, n. 1, p. 87-95, ene. 2010.

AROCA, A.; BLANCO-ÁLVAREZ, H.; GIL CHAVES, D. Etnomatemática y formación inicial de profesores de matemáticas: el caso colombiano. Revista Latinoamericana de Etnomatemática, Colombia, v. 9, n. 2, p. 85-102, jun. 2016.

BALL, D. L. Bridging practices: Intertwining content and pedagogy in teaching and learning to teach. Journal of Teacher Education, Estados Unidos, v. 51, p. 241-247, mayo. 2000.

BALL, D. L.; THAMES, M. H.; PHELPS, G. Content knowledge for teaching: What makes it special? Journal of Teacher Education, Estados Unidos, v. 59, n. 5, p. 389-407, 2008.

BARDIN, L. Análisis de contenido. 2. ed. Madrid: Ediciones Akal, 1996. 192 p.

BISHOP, A. Educando a los «culturizadores matemáticos». Uno: Revista de Didáctica de las Matemáticas, España, v. 6, p. 7-12, oct. 1995.

BLANCO-ÁLVAREZ, H. La postura sociocultural de la educación matemática y sus implicaciones en la escuela. Revista Educación y Pedagogía, Colombia, v. 23, n. 59, p. 59-66, ene. 2011.

BLANCO-ÁLVAREZ, H. Estudio de las actitudes hacia una postura sociocultural y política de la Educación Matemática en profesores en formación inicial. REDIMAT - Journal of Research in Mathematics Education, Barcelona, v. 1, n. 1, p. 57-78, feb. 2012.

BLANCO-ÁlVAREZ, H.; HIGUITA RAMÍREZ, C.; OLIVERAS, M. L. Una mirada a la Etnomatemática y la Educación Matemática en Colombia: caminos recorridos. Revista Latinoamericana de Etnomatemática, Colombia, v. 7, n. 2, p. 245-269, jun. 2014.

BREDA, A.; DO ROSÁRIO, V. M. Etnomatemática sob dois pontos de vista: a visão D“Ambrosiana e a visão Pós-Estruturalista. Revista Latinoamericana de Etnomatemática, Colombia, v. 4, n. 2, p. 431, ago. 2011.

BREDA, A.; DO ROSÁRIO LIMA, V. M.; DURO, G. T. A. Etnomatemática nos cursos de formação continuada de professores: implicações das regularidades discursivas e das relações de poder na produção de subjetividades. Revista Latinoamericana de Etnomatemática, Colombia, v. 5, n. 1, p. 116-148, feb. 2012. 
CARRILLO, J. Building mathematical knowledge in teaching by means of theorised tools. In: ROWLAND, T.; RUTHVEN, K. (Ed.). Mathematical Knowledge in Teaching. New york: Ed. Springer, 2011. p. 273-287.

CARRILLO, J.; CONTRERAS, L. C.; FLORES, P. Un modelo de conocimiento especializado del profesor de matemáticas. In: RICO, L. et al. (Ed.). Investigacion en Didáctica de la Matemática. Homenaje a Encarnación Castro. Granada: Editorial Comares, 2013. p. 193-200.

CLEMENTS, M. A et al. (Ed.). Third International Handbook of Mathematics Education. 1. ed. New York: Ed. Springer, 2013. 1120 p.

D’AMBROSIO, U. Etnomatemática. Elo entre as tradições e a modernidades. 5. ed. Brasil: Autêntica Editora, 2005. 112 p.

D’AMBROSIO, U. Las bases conceptuales del Programa Etnomatemática. Revista Latinoamericana de Etnomatemática, Colombia, v. 7, n. 2, p. 100-107, jun. 2014.

DÍEZ-PALOMAR, J. La formación de matemáticas para las familias. Una mirada desde la etnomatemática. Revista Latinoamericana de Etnomatemática, Colombia, v. 4, n. 2, p. 55-69, ago. 2011.

DOMITE, M. C. The encounter of non-indigenous teacher educator and indigenous teacher: the invisibility of the challenges. ZDM, Alemania, v. 42, n. 3-4, p. 305-313, abr. 2010.

DOMITE, M. C. Acertando o passo do movimento entre etnomatemática, formação de professores e aprendizagem da matemática: pré-requisito dos alunos e escuta dos professores em discussão. Revista Educação Matemática em Foco, Campina Grande, v. 1, n. 1, p. 83-96, ene./jun. 2012.

FERNÁNDEZ-OLIVERAS, A.; OLIVERAS, M. L. Formación de profesores y Microproyectos curriculares. Revista Latinoamericana de Etnomatemática, Colombia, v. 8, n. 2, p. 472-495, jun. 2015.

FOUCAULT, M. 1. ed. Microfísica do poder. Rio de Janeiro: Ed. Graal, 1979. 163 p.

FOUCAULT, M. História da Sexualidade I: a vontade de saber. 14. ed. Rio de Janeiro: Ed. Graal, 2001. 149 p.

FOUCAULT, M. Arqueologia do saber. 6. ed. Rio de Janeiro: Ed. Forense Universitária, 2002. 236 p.

FOUCAULT, M. A ordem do discurso. 15. ed. São Paulo: Ed. Loyola, 2007. 79 p.

FREIRE, P. Pedagogia da Autonomia: saberes necessários à prática educativa. 1. ed. São Paulo: Ed. Paz e Terra, 1996. 144 p.

GAVARrete, M. E. Modelo de aplicación de Etnomatemáticas en la Formación de Profesores para Contextos Indígenas de Costa Rica. 2012. 734 f. Tesis (Doctorado en Educación) -Universidad de Granada, España. 2012. No publicado.

GAVARRETE, M. E. La Etnomatemática como campo de investigación y acción didáctica: su evolución y recursos para la formación de profesores desde la equidad. Revista Latinoamericana de Etnomatemática, Colombia, v. 6, n. 1, p. 127-149, feb. 2013. 
GAVARRETE, M. E. Etnomatemáticas indígenas y formación docente: una experiencia en Costa Rica a través del modelo MOCEMEI. Revista Latinoamericana de Etnomatemática, Colombia, v. 8, n. 2, p. 136-176, jun. 2015.

GAVARRETE, M. E.; ALBANESE, V. Etnomatemáticas de signos culturales y su incidencia en la formación de profesores. Revista Latinoamericana de Etnomatemática, Colombia, v. 8, n. 2, p. 299-315, jun. 2015.

GAVARRETE, M. E.; OLIVERAS, M. L. Matemáticas, culturas y formación de profesores en Costa Rica. Journal of Mathematics and Culture, Estados Unidos, v. 6, n. 1, p. 209-222, mar. 2012.

GERDES, P. Etnomatemática e educação matemática: uma panorâmica geral. Revista Quadrante, Portugal, v. 5, n. 2, p. 105-138, jul. 1996.

GERDES, P. On culture and mathematics teacher education. Journal of Mathematics Teacher Education, Estados Unidos, v. 1, n. 1, p. 33-53, ene. 1998.

GERDES, P. Exploration of technologies, emerging from African cultural practices, in mathematics (teacher) education. ZDM, Alemania, v. 42, n. 1, p. 11-17, feb. 2010.

GERDES, P. Reflexões sobre o ensino da matemática e diversidade cultural. Revista Latinoamericana de Etnomatemática, Colombia, v. 7, n. 2, p. 108-118, jun. 2014.

GIL, A. C. Métodos e técnicas de pesquisa social. 5. ed. São Paulo: Ed. Atlas, 1999. 200 p.

GODINO, J. D. et al. Componentes e indicadores de idoneidad de programas de formación de profesores en didáctica de las matemáticas. Revemat: revista eletrônica de educação matemática, Brasil, v. 8, n. 1, p. 46-74, jul. 2013.

GOLDIN, G. A. et al. Beliefs and engagement structures: behind the affective dimension of mathematical learning. ZDM, Alemania, v. 43, n. 4, p. 547-560, jul. 2011.

JAWORSKI, B. Mathematics teaching development as a human practice: identifying and drawing the threads. ZDM, Alemania, v. 44, n. 5, p. 613-625, jun. 2012.

KATSAP, A.; SILVERMAN, F. L. A Case Study of the Role of Ethnomathematics among Teacher Education Students from Highly Diverse Cultural Backgrounds. Journal of Mathematics and Culture, Estados Unidos, v. 3, n. 1, p. 66-102, jun. 2008.

KNIJNIK, G.; MEREGALLI, J. Educação matemática em cursos de Pedagogia: um estudo com professores brasileiros dos anos iniciais de escolarização. Revista Latinoamericana de Etnomatemática, Colombia, v. 5, n. 2, p. 4-20, nov. 2012.

LADSON-BILLINGS, G. But that's just good teaching! The case for culturally relevant pedagogy. Theory Into Practice, v. 34, n. 3, p. 159-165, jun. 1995.

MADUSISE, S. Cultural villages as contexts for mediating culture and mathematics education in the South African curriculum. Revista Latinoamericana de Etnomatemática, Colombia, v. 8, n. 2, p. 11-31, jun. 2015.

MARTÍNEZ, O. J. Una experiencia de capacitación en etnomatemática, en docentes Indígenas venezolanos. Journal of Mathematics and Culture, Estados Unidos, v. 6, n. 1, p. 286-295, mar. 2012. 
MARTÍNEZ, O. J.; OLIVERAS, M. L. Surcando caminos de interculturalidad sustentados en la Etnomatemática. Revista Latinoamericana de Etnomatemática, Colombia, v. 8, n. 2, p. 341-363, jun. 2015.

MENDES, I. A. O estudo da realidade como eixo da formação matemática dos professores de comunidades rurais. BOLEMA: Boletim de Educação Matemática, Rio Claro, v. 23, n. 36, p. 571$595,2010$.

MONTEIRO, A.; RODRIGUES MENDES, J. R. A etnomatemática no encontro entre práticas e saberes: Convergências, tensões e negociação de sentidos. Revista Latinoamericana de Etnomatemática, Colombia, v. 7, n. 3, p. 55-70, oct. 2014.

MOREIRA, D. A etnomatemática e a formação de professores. Discursos: perspectivas em educação. Lisboa, n. 2. p. 27-38, dic. 2004

MORGAN, C. Assessment of mathematical behaviour: A social perspective. In: GATES, P. (Ed.). MATHEMATICS, EDUCATION AND SOCIETY CONFERENCE (MEAS 1), 1., 1998, Inglaterra. Anais...Nottingham: Nottingham University, 1998. p. 227-283.

NARESH, N. The role of a critical ethnomathematics curriculum in transforming and empowering learners. Revista Latinoamericana de Etnomatemática, Colombia, v. 8, n. 2, p. 450-471, jun. 2015.

OLIVERAS, M. L. Artesanía andaluza y matemáticas. Un trabajo transversal con futuros profesores. Uno: Revista de Didáctica de las Matemáticas, España, n. 6, p. 73-84, oct. 1995.

OLIVERAS, M. L. Etnomatemáticas: formación de profesores e innovación curricular. 1. ed. Granada: Ed. Comares, 1996. 133 p.

OLIVERAS, M. L. Ethnomathematics and Mathematical Education. ZDM, Alemania, v. 31, n. 3, p. 891, jun. 1999.

OLIVERAS, M. L. Microproyectos para la educación intercultural en Europa. Uno: Revista de Didáctica de las Matemáticas, España, n. 38, p. 70-81, ene. 2005.

OLIVERAS, M. L. Etnomatemáticas. De la multiculturalidad al mestizaje. In: GIMENEZ, J.; GOÑI, J. M.; GUERRERO, S. (Eds.). Matemáticas e interculturalidad. Barcelona: Graó, 2006. p. 117-149.

OLIVERAS, M. L.; GAVARRETE, M. E. Modelo de aplicación de etnomatemáticas en la formación de profesores para contextos indígenas en Costa Rica. RELIME: Revista Latinoamericana de Investigación en Matemática Educativa, México, v. 15, n. 3, p. 339-372, nov. 2012.

OWENS, K. Identity as a Mathematical Thinker. Mathematics Teacher Education and Development (MTED), Nueva Zelanda, v. 9, p. 36-50, 2007.

OWENS, K. The impact of a teacher education culture-based project on identity as a mathematically thinking teacher. Asia-Pacific Journal of Teacher Education, Inglaterra, v. 42, n. 2, p. 186-207, mar. 2014.

PINO-FAN, L.; GODINO, J. D. Perspectiva ampliada del conocimiento didáctico-matemático del profesor. Paradigma, Venezuela, v. 36, n. 1, p. 87-109, 2015.

PONTE, J. P. Mathematics teacher education programs: practice and research. Journal of Mathematics Teacher Education, Netherlands, v. 15, n. 5, p. 343-346, oct. 2012. 
PONTE, J. P. Estudiando el conocimiento y el desarrollo profesional del profesorado de matemáticas. In: PLANAS, N. (Ed.). Teoría, crítica y práctica de la educación matemática Barcelona: Ed. Graó, 2013. p. 93-98.

PRESMEG, N. C. Ethnomathematics in Teacher Education. Journal of Mathematics Teacher Education, Netherlands, v. 1, n. 3, p. 317-339, oct. 1998.

RAMOS, E. Reflexión docente sobre la enseñanza del álgebra en un curso de formación continua. 2014. 353 f. Tesis (Doctorado en Educación) - Facultad de Ciencias de la Educación, Universidad de Granada, España, 2014.

REZAT, S.; STRÄSSE, R. From the didactical triangle to the socio-didactical tetrahedron: artifacts as fundamental constituents of the didactical situation. ZDM, Alemania, v. 44, n. 5, p. 641-651, ago. 2012.

RODRIGUES, M.; FERREIRA, F.; DOMITE, M. C. A formação de professores e suas relações com cultura e sociedade: a educação escolar indígena no centro das atenções. Bolema, Rio Claro, v. 22, n. 34 , p. 263-282, 2009.

ROJAS, N. Caracterización del conocimiento especializado del profesor de matemáticas: un estudio de casos. 2014. 335 f. Tesis (Doctorado en Educación) - Facultad de Ciencias de la Educación, Universidad de Granada, España, 2014.

SANDÍN, M. P. Investigación cualitativa en educación: fundamentos y tradiciones. 1. ed. Madrid: McGraw-Hill, 2003. 258 p.

SANTILLÁN, A. Aportes para la construcción de una historia de la matemática: Experiencia en el profesorado de matemática en la Universidad Nacional del Chaco Austral, Argentina. Revista

Latinoamericana de Etnomatemática, Colombia, v. 4, n. 1, p. 40-54, feb. 2011.

SANTILLÁN, A.; ZACHMAN, P. Una experiencia de capacitación en Etnomatemática. Revista Latinoamericana de Etnomatemática, Colombia, v. 2, n. 1, p. 27-42, feb. 2009.

SCHÖN, D. Educating a Reflexive Practitioner. Toward a New Design for Teaching and Learning in the Professions. 1. ed. São Francisco: Jossey-Bass, 1987. 376 p.

SCHÖN, D. Formar professores como profissionais reflexivos. In: NOVOA, A. (Org.). Os professores e a sua formação. Lisboa: Ed. Dom Quixote, 1995. p. 77-91.

SHIRLEY, L. Ethnomathematics as a Fundamental of Instructional Methodology. ZDM, Alemania, v. 33, n. 3, p. 85-87, jun. 2001.

SHULMAN, L. S. Those Who Understand: Knowledge Growth in Teaching. Educational Researcher, Estados Unidos, v. 15, n. 2, p. 4-14, feb. 1986.

STAKE, R. E. Investigación con estudio de casos. 2. ed. Madrid: Ediciones Morata, 1998. 155 p.

STRAUSS, A.; CORBIN, J. Bases de la investigación cualitativa. Técnicas y procedimientos para desarrollar la teoría fundamentada. 1. ed. Medellín: Editorial Universidad de Antioquia. 2002. 354 p.

TALL, D. Using the computer as an environment for building and testing mathematical concepts: A tribute to Richard Skemp. In: Papers in Honour of Richard Skemp. Warwick: [s.n.], 1986. p. 21-36. 
THOMPSON, A. Teacher's Beliefs and Conceptions: A Synthesis of the Research. In: GROUWS, D. (Ed.). Handbook of Research on Mathematics Teaching and Learning. New York: Ed. MacMillan Publishing Company, 1992. p. 127-146.

TIROSH, D.; WOOD, T. (Ed.). 1. ed. The International Handbook of Mathematics Teacher Education. Rotterdam: Sense Publishers, 2008. 330 p.

VALERO, P. La Educación Matemática como una red de prácticas sociales. In: VALERO, P.; SKOVSMOSE, O. (Ed.). Educación Matemática Crítica: Una visión sociopolítica del aprendizaje y la enseñanza de las matemáticas. Bogotá: Ed. Ediciones Uniandes, 2012. p. 298-326.

VERNER, I.; MASSARWE, K.; BSHOUTY, D. Constructs of engagement emerging in an ethnomathematically-based teacher education course. The Journal of Mathematical Behavior, Estados Unidos, v. 32, n. 3, p. 494-507, sep. 2013.

WITTGENSTEIN, L. Investigaciones filosóficas. 1. ed. Barcelona: Ediciones Altaya, 1999. 126 p.

Submetido em Abril de 2016. Aprovado em Março de 2017. 